\title{
Quiste aracnoideo sacro recidivante con meningocele anterior gigante. Reporte de caso
}

Nathalia Jácome-Pérez ${ }^{\star}$ Feligno Barliza Quintero², Oscar Leonardo Torra-Barajas³ , Luis Eduardo Franco-Ospina4 .

1. Universidad Industrial de Santander. Bucaramanga. Santander. Colombia.

2. Universidad del Magdalena. Santa Marta. Magdalena. Colombia.

3. Universidad Autónoma de Bucaramanga. Bucaramanga. Santander. Colombia.

4. Radiólogo. Universidad Militar de Colombia. Hospital Universitario de Santander. Docente Universidad Industrial de Santander. Bucaramanga. Santander. Colombia.

\section{Sacral arachnoid recurrent cyst with giant anterior meningocele, a case report}

Resumen: Los quistes aracnoideos corresponden a lesiones benignas expansivas del canal medular secundarias a defectos anatómicos durales, mientras que los meningoceles anteriores consisten en la herniación de la duramadre hacia la pelvis a través de forámenes dilatados o defectos óseos. Ambas entidades son infrecuentes y sus manifestaciones clínicas puede variar de acuerdo a estructuras anatómicas que comprimen. Una historia clínica completa, la pesquisa diagnóstica y la adecuada interpretación de imágenes orientan al diagnóstico y manejo de estos pacientes. Se presenta el caso de una paciente adulta mayor con historia de masa pélvica, dolor lumbar severo y monoparesia en quien se realizó el diagnóstico incidental de quiste aracnoideo sacro y meningioma sacro anterior.

Palabras clave: Imagen por resonancia magnética, Quistes aracnoideos, Meningocele.

Abstract: Arachnoid cysts are benign expansive lesions of the spinal canal secondary to dural defects, whereas the anterior meningoceles consist of the herniation of the dura into the pelvis through dilated foramina or bone defects. Both pathologies are infrequent and its clinical manifestations vary according to compressed anatomical structures. A complete clinical history, the diagnostic investigation and the correct imaging studies interpretation guide the diagnosis and management of these patients. We present the case of an elderly adult patient with a history of pelvic mass, severe lumbar pain and monoparesis in whom the incidental diagnosis of sacral arachnoid cyst and anterior sacral meningioma was performed Key words: Arachnoid cysts, Magnetic resonance imaging, Meningocele.

Jácome $N$, et al. Quiste aracnoideo sacro recidivante con meningocele anterior gigante, reporte de caso. Rev Chil Radiol 2019; 25(2): 75-78.

*Correo electrónico: Nathalia Jácome Pérez / njpdra@hotmail.com

Trabajo enviado el 22 de abril de 2019. Aceptado para publicación el 29 de mayo de 2019

\section{Introducción}

Los quistes aracnoideos espinales corresponden a entidades infrecuentes, que se definen como "divertículos" que contienen líquido cefalorraquídeo (LCR) y se extienden al espacio extradural ${ }^{(1)}$. Se presentan en su mayoría en la región torácica baja con un $65 \%$, seguido de la región lumbar con el $13 \%{ }^{(2)}$. Incluidos en este grupo se describe el meningocele sacro anterior, que corresponde a una extensión de la duramadre y la aracnoides fuera del canal espinal del sacro hacia el retroperitoneo y espacio intraperitoneal a través de un defecto sacro ventral(3). Estas lesiones expansivas pueden manifestarse clínicamente con dolor axial y síntomas relacionados con la compresión del cordón o la raíz ${ }^{(4)}$.

En este artículo se describe una paciente con quiste aracnoideo extradural que fue manejado quirúrgicamente en varias ocasiones sin éxito, en quien además se encontró la presencia de lesión quística compatible con meningocele sacro anterior gigante. 


\section{Presentación de caso}

Mujer de 66 años, quien consultó al año 2012 por una historia de dos años de dolor en región lumbosacra. Una tomografía computarizada (TAC) de columna lumbosacra mostró una dilatación del canal raquídeo desde el cuerpo vertebral L4 hasta el plano sacrocoxigeo con múltiples imágenes hipodensas de aspecto sacular en el espacio presacro, asociado a prominentes quistes radiculares, considerándose aracnoidocele vertebral. Se realizó laminectomía lumbosacra y descompresión del canal medular desde el cuerpo vertebral L5 a S3, con posterior aparición de fistula de LCR.

La paciente persistió con dolor radicular lumbar acompañado de monoparesia derecha. Una nueva TAC el año 2013 muestra aumento de volumen del canal raquídeo y de los forámenes de conjunción secundaria a lesión quística con remodelación ósea, grandes dilataciones quísticas de las vainas radiculares compatible con un meningocele lumbosacro gigante que fue tratado con una derivación cistoperitoneal de lesión.

El año 2018 consulta por recidiva del dolor, masa suprapúbica blanda, dolorosa a la palpación no móvil de aproximadamente $7 \times 10 \mathrm{~cm}$, y monoparesia inferior izquierdo. Un estudio de TAC y RMN mostró lesión quística presacra conectada al espacio subaracnoideo, con ensanchamiento del saco tecal y remodelación ósea de muros posteriores de los cuerpos vertebrales lumbosacros, con el catéter de la deriviación cistoperitoneal in situ (Figuras 1, 2 y 3). Se consideró drenaje de la lesión y clampeo del catéter de derivación por laparoscopia sin embargo la paciente solicitó salida voluntaria.
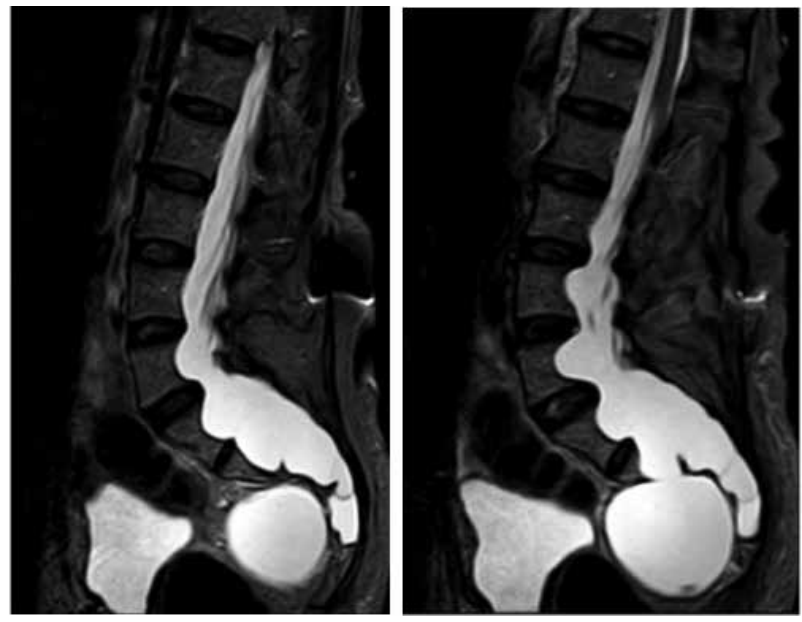

Figura 1: Cortes sagitales de RMN secuencia T2 en los que se aprecia una ectasia dural lumbosacra con remodelación de los contornos de los cuerpos vertebrales. Adicionalmente se observa una lesión quística presacra que se comunica con el canal.
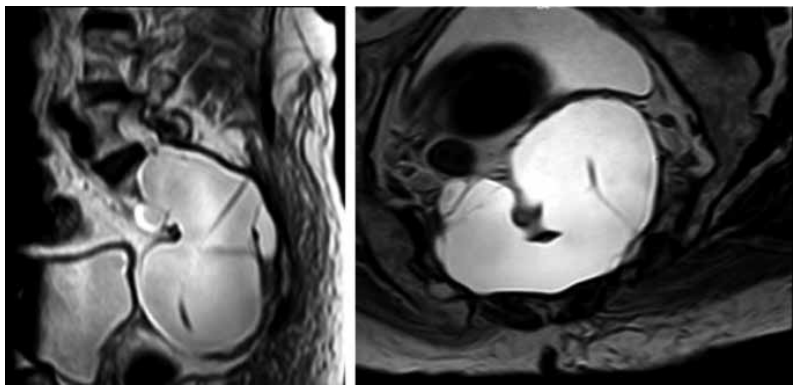

Figura 2: Cortes axiales y sagitales de una RMN con secuencias T2. Lesión quística intrarraquídea con extensión pre sacra y catéter de derivación in situ, correspondiente a menigocele sacro anterior recidivado.
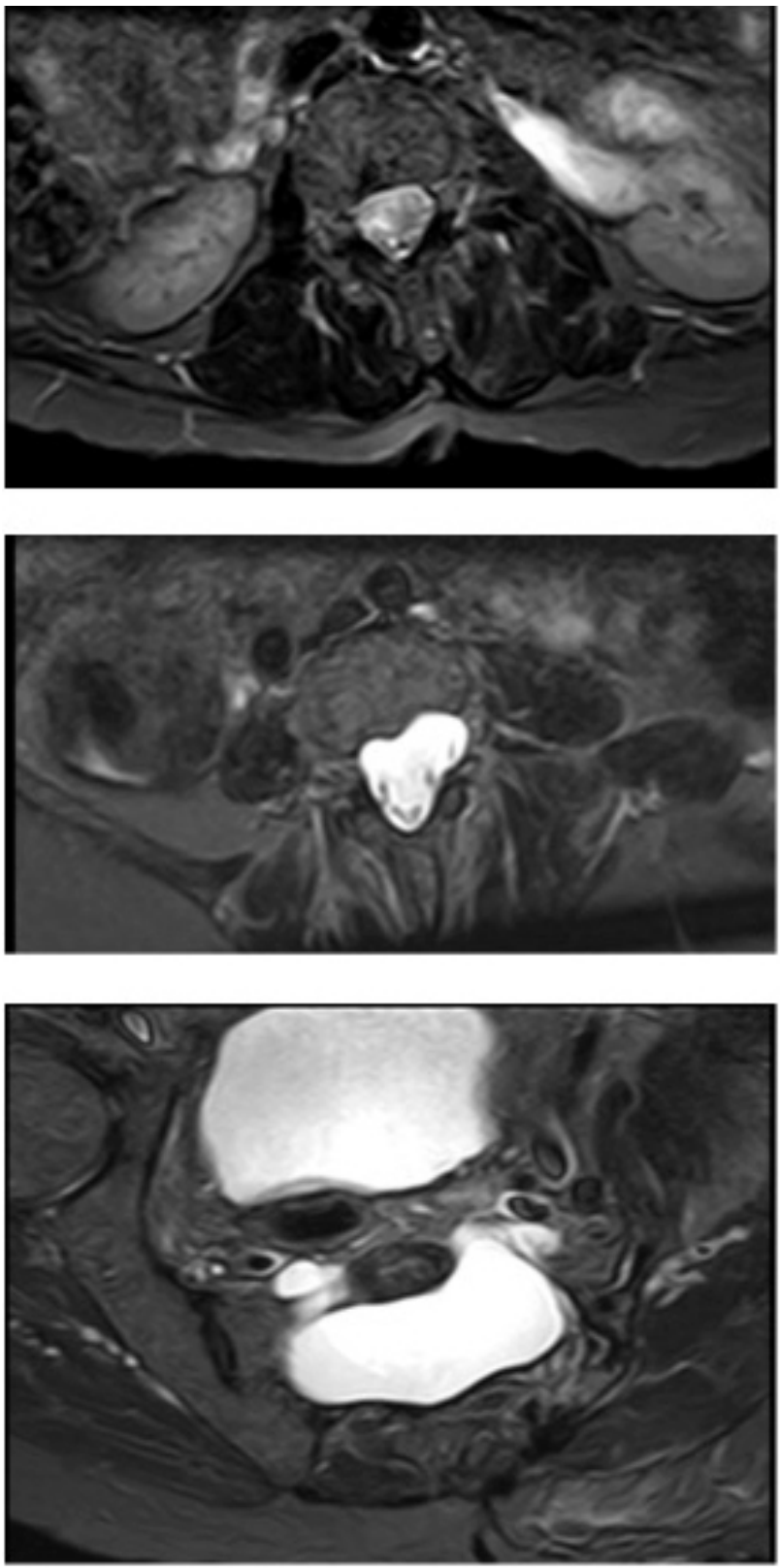

Figura 3: RMN, secuencia T2, que muestra progresiva del canal raquídeo por el quiste aracnoideo intrarraquídeo conocido. 


\section{Discusión}

Los quistes extradurales se desarrollan a partir de protrusiones de la membrana aracnoidea a través de un defecto dural que contienen LCR, y permiten la herniación de la misma; estos tienen un pedículo en el defecto dural que comunica el espacio subaracnoideo espinal con el espacio extradural(5). Son más frecuentes en hombres durante la segunda década y tienden a ser asintomáticos ${ }^{(1)}$. Su etiología no es clara aún, si bien una de las hipótesis más aceptadas explica que se forman por alteraciones fibróticas en el espacio subaracnoideo ${ }^{(6)} \mathrm{O}$ adherencias que dan lugar a zonas lobuladas con LCR en su interior secundarias a inflamación o infección ${ }^{(1,6)}$.

Algunas teorías mencionan que el revestimiento celular en las paredes del quiste tiene capacidades secretoras de líquido, lo que explicaría su crecimiento hacia el espacio extradural ${ }^{(1,2,7)}$. Otros autores defienden la existencia de un mecanismo de "válvula" unidireccional que permitenel crecimiento del quiste por los incrementos intermitentes de presión en el $\operatorname{LCR}^{(1,3,6)}$. Los síntomas a menudo son asimétricos e incluyen debilidad, dolor neuropático, mielopatía, incontinencia, infecciones del tracto urinario y paraparesia ${ }^{(2,4)}$.

Los meningoceles sacros corresponden a herniacionen de la duramadre hacia la pelvis a través de defectos preexistentes en los agujeros sacros o forámenes de conjución. Su mayor incidencia es entre 20 - 39 años de edad(8). A diferencia de los quistes aracnoideos, los meningoceles sacros anteriores se presentan en su mayoría en mujeres ${ }^{(9)}$.

La sintomatología secundaria al meningocele sacro anterior está directamente relacionada con las estructuras y raíces nerviosas que comprima abarcando desde dolor lumbar crónico (siendo el principal síntoma) hasta estreñimiento, incontinencia urinaria, sintomatología ginecológica, descritas en un $10 \%$ de los casos de acuerdo a Oren $M$ et al ${ }^{(9)}$. De acuerdo a Hollenberg et $\mathrm{al}^{(10)}$, el hallazgo de una masa palpable puede acompañarse de los síntomas antes descritos.

La RMN sigue siendo el estudio de elección para el diagnóstico de disrafismos espinales, puede complementarse con la mielografía para determinar el contenido del saco herniario. Además de caracterizar la lesión, permite clasificar y planear el abordaje quirurgico. Los meningoceles sacro anterior en las secuencias en RMN se describen como una lesión quística con señal de LCR, rodeado de una fina pared perceptible ${ }^{(11)}$.

Los quistes aracnoideos, en RMN corresponden a una lesión quística hipointensa en secuencias T1, e hiperintensa en secuencias T2, de localización intrarraquídea que se extiende a los foramenes neurales. Ante sintomatología persistente o progresión de déficit neurológico se indica manejo quirúrgico en donde el objetivo será la descompresión neural y evitar la reexpansión del quiste. Se han descrito técnicas como resección, fenestración o derivaciones de acuerdo a las características del quiste y su localización. Adicionalmente se recomienda realizar una hemilaminectomia en quistes aracnoideos grandes con el fin de reducir los riesgos de complicaciones postquirúrgicas ${ }^{(12)}$. En este caso, dado el tamaño del defecto se realizó laminectomia lumbosacra con descompresión del canal raquídeo con posterior aparición de una fistula de LCR, una de las complicaciones más frecuentes descritas.

En resumen, los quistes aracnoideos sacros y los meningoceles sacro anterior son entidades infrecuentes. La presencia de dolor lumbar crónico o déficits sensitivos en miembros inferiores de larga data pueden sospechar su diagnóstico. Sin embargo, existe una variedad de sintomatologías posibles secundaria a las estructuras que comprima. Una historia clínica completa, la pesquisa diagnóstica y la adecuada interpretación imagenológica permiten la correcta caracterización de estas lesiones.

\section{Consideraciones éticas}

El presente trabajo corresponde a un reporte de caso por lo que no implica riesgo inherente a la intervención en los individuos incluidos. Según lo que establece la resolución colombiana clasifica como un estudio sin riesgo pues no realiza ninguna intervención o modificación intencional en ninguna variable sobre el paciente incluido. Se solicitó consentimiento informado al paciente quien aceptó la publicación del caso para fines científicos.

\section{Conflictos de interés}

Los autores declaran no tener conflictos de interés

\section{Autoría}

1. Responsables de la integridad del estudio: NJP, FBQ, OLTB.

2. Concepción del estudio: NJP, FBQ, LEFO

3. Diseño del estudio: NJP, FBQ, LEFO

4. Obtención de los datos: NJP, FBQ

5. Análisis e interpretación de los datos: NJP, FBQ

6. Tratamiento estadístico: no procede.

7. Búsqueda bibliográfica: NJP, OLTB.

8. Redacción del trabajo: NJP, FBQ, OLTB.

9. Revisión crítica del manuscrito con aportaciones intelectualmente relevantes: LEFO

10. Aprobación de la versión final: NJP, FBQ, OLTB, LEFO.

\section{Referencias}

1. Shedidd D, Rogerp EP, Banzelc EC. Presacral meningocele: diagnosis and treatment. Semin SpinegSurg. 2006; 18: 161-167.

2. Leew SW, Fooa A, Tanl CL, Tant T, Iwins S, Yeot TT, et al. Spinal extradural cyst: A case report and review of literature. World neurosurgery.2018. Doi: 10.1016/ j.wneu.2018.05.199. 
3. Guptas S, Chunnilalj J, Mehrotram M, Mehrotraa A, Srivastavak AK, Dask KK. Recurrent abortion and tethered cord syndrome due to anterior sacral meningocele: $A$ report of rare case with review of literature. World neurosurgery. 2017. Doi: 10.1016/j.wneu.2017.02.112.

4. Dahlgreenm RM, Baronm EM, Vaccaroa A. Pathophysiology, diagnosis and treatment of spinal meningoceles and arachnoid cysts. Semin spin surg. 2006; 18: 148-153.

5. Maenhoudtw W, Rasschaertr R, Bontinckh H, Pinsonh $H$, van Roostd D, Hallaertg G. Post-arachnoiditis anterior spinal arachnoid cyst formation with compressive myelopathy: report of 2 cases. World neurosurgery. 2018. Doi: 10.1016/j.wneu.2018.07.006.

6. Hernándezo O, Pérezf F, Corralesn N. Postraumatic epidural arachnoid spinal cyst: Case report. Neurocirugía. 2011; 22: 267-270.

7. Viswanathanv V, Manoharanr SR, Doh H, Minnemaa A, Shaddym SM, Elderb JB, et al. Clinical and radiologic outcomes following fenestration and partial wall excision of idiopathic intradural spinal arachnoid cysts presenting with myelopathy. World neurosurgery. 2017.
Doi: 10.1016/j.wneu.2017.05.136.

8. Villarejof F, Scavonec C, Blazquezg MG,PascualCastroviejoil, Perez Higuerasa A, Fernandez-Sanchez A, et al. Anterior sacral meningocele: Review of the literature. Surg neurol. 1983; 19: 57-71.

9. Orenm M, Laberb B, Leeh SH. Anterior sacral meningocele. Report of five cases and review of the literature. Dis colon rectum. 1977; 20: 492.

10. Hollenbergm AM, Mesfina A, Silbersteinh H. Rupture of giant anterior sacral meningocele in a patient with marfan syndrome: Diagnosis and management. World neurosurg. 2018; 119: 137-141.

11. QijJ, Yangj J, Wangh GH. A novel five-category multimodal T1-weighted and T2-weighted magnetic resonance imaging-based stratification system for the selection of spinal arachnoid cyst treatment: A 15-year experience of 81 cases. Neuropsychiatr dis treat. 2014; 10: 499-506.

12. Leej HJ, Choh WH, Hanh IH, Choik BK. Large thoracolumbar extradural arachnoid cyst excised by minimal skipped hemilaminectomy: A case report. Koreanj JeSpine. 2013; 10: 28-31. 